CCXXVI.-Harmine and Harmaline. Part VI. The Synthesis of N-Methyltetrahydronorharmine and the Constitution of Harmaline and of the Alkylated Harmines.

By William Oailvy Kermack, Winliam Henry Perkin, and ROBERT ROBINSON.

IT has been clearly shown in the earlier sections of this investigation * that the analytic evidence which may be brought to bear on the problem of the constitution of harmine indicates that this alkaloid should be represented by the expression $I$, and the correctness of this view has been confirmed by the syntheses of norharman (II) and of harman (III), which may be derived from the base by simple processes of degradation.

(I.)<smiles>COCC1NC2CC3CC(C2)C1N3</smiles>

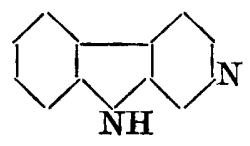

* Part I, T., 1912, 101, 1775; Part II, T., 1913, 103, 1973; Part III, T., 1919, 115, 933; Part IV, T., 1919, 115, 967; Part V, T., 1921, 119, 1602. 
HARMINE AND HARMALINE. PART VI. View Article Online

(III.)
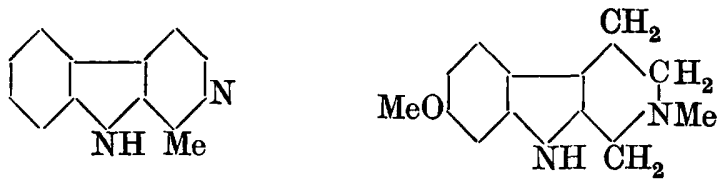

In the present communication, we are able to describe the synthesis of derivatives containing the methoxy-group, the establishment of the position of which by such means removes any possible remaining doubt of the accuracy of the formula which has been put forward as a summary of the reactions of harmine. In order to enable a comparison to be made with the harmine and harman formulæ, it may be stated at once that the substance which we have synthesised is $N$-methyltetrahydronorharmine (IV), which may be prepared from either harmine or harmaline by methods detailed in the sequel.

The trinuclear system has been built up by taking advantage of the process developed in connexion with the synthesis of norharman (Part V). Essentially this consists in the ring closure of an acetalylamide of an indole-2-carboxylic acid under the influence of alcoholic hydrochloric acid. The complication which arises from the fact that the elimination of the elements of two molecules of alcohol can occur in two directions is illustrated in the following examples already described in Part V (pp. 1607, 1611).

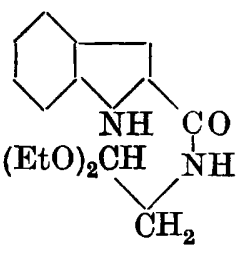

$(\mathrm{EtO})_{2} \mathrm{CH}$

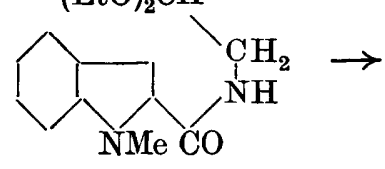

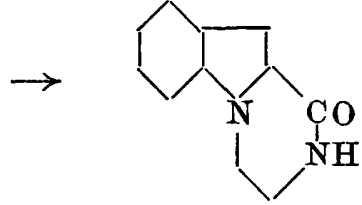

Ketodihydroindolediazine.

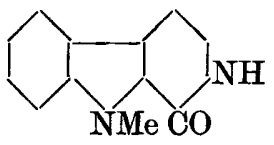

(V.)

We were thus able to prepare 1-methylcarboline derivatives, and V, on distillation over zinc dust, was converted into norharman. The yield was, however, poor and the purification difficult, and we were very reluctant to contemplate the necessity for the employment of such a method in the synthesis of harmine or norharmine. Moreover, there are grave disadvantages attaching to the application of the zinc dust distillation process to methoxy-compounds, and a

vor. CXXI. 
good instance of the anomalous results which may be anticipated is furnished by the work of Irvine and Moodie (T., 1907, 91, 536) on the nature of the substances obtained by distilling $o$-dimethoxybenzoin over zine in a stream of hydrogen. In this reduction the main product was $p$-dimethyltolane. In any case it appeared to us that considerable difficulty must be experienced in removing the alkyl group attached to the pyrrole-nitrogen atom, and we therefore attempted the preparation of carboline derivatives from indole-2-carboxyacetalylamides which contain an unsubstiuted imino-group in the indole nucleus. The fact that the reactions leading to the indolediazine and carboline types both occurred with facility was encouraging, and our expectation that the balance between the ring closure in the two directions might be a somewhat delicate one was strikingly confirmed by the first modification attempted. Whereas indole-2-carboxyacetalylamide gives, as depicted above, an indolediazine, indole-2-carboxydimethylacetalylmethylamide (VI) yields a ketomethyldihydrocarboline (VII) on treatment with alcoholic hydrochloric acid.

(VI.)
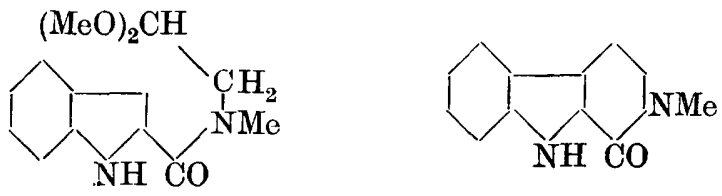

(VII.)

It is very remarkable that such a small constitutional change should so completely alter the course of the reaction and it is difficult to suggest a satisfactory explanation. On distillation over zinc dust in a stream of hydrogen, the new ketomethyldihydrocarboline yields indole and a small amount of norharman. The conversion of 6-methoxyindole-2-carboxyacetalylamide (VIII) nto the indolediazine derivative (IX) has already been described (Part V, p. 1613), and it was of great interest to determine whether in this series also a carboline would result if the amide nitrogen is alkylated.

(VIII.)
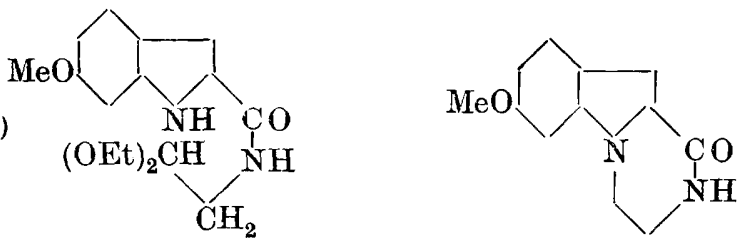

We accordingly prepared 6-methoxyindole-2-carboxydimethylacetalylmethylamide $(\mathrm{X})$ and studied its behaviour towards alcoholic hydrochloric acid. In this case, two substances, $\mathrm{C}_{\mathbf{1 3}} \mathrm{H}_{\mathbf{1 2}} \mathrm{O}_{\mathbf{2}} \mathrm{N}_{\mathbf{2}}$, 
HARMINE AND HARMALINE. PART VI. View Article Online

were isolated and comparison with undoubted indolediazines and carbolines showed beyond question that, of these, the substance melting at $246^{\circ}$ is methoxyketomethyldihydrocarboline (XI) and the isomeride melting at $205^{\circ}$ is methoxykctomethyldihydroindolediazine (XII).

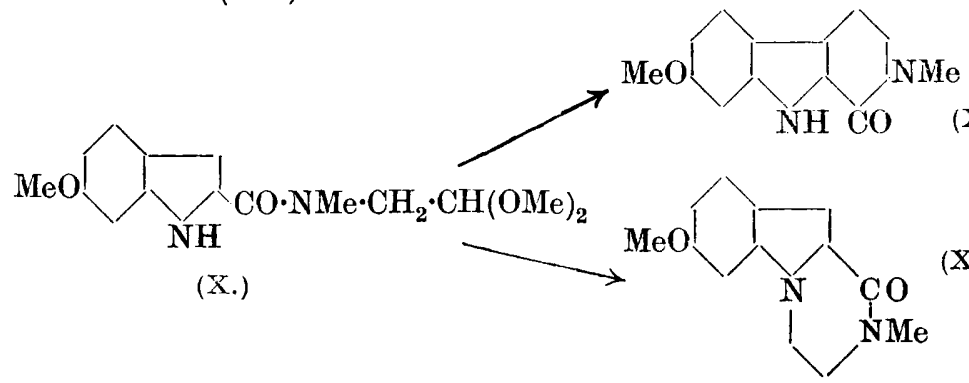

The influence of the methyl attached to the amidic nitrogen is thus again observed, but a comparison with the former example indicates that the methoxyl group has tended to favour the formation of the indolediazine derivative. This is probably equivalent to the statement that the methoxyl group has activated the hydrogen in position $l$ in the indole nucleus to a greater extent than that in position 3. The following scheme illustrates the effect of the negative oxygen atom on the hydrogen atoms in question, and the results observed are seen to be in agreement with the theory of alternate polarity of atoms in a chain.

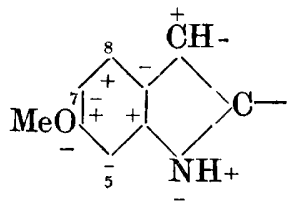

Plainly, methoxyl in position 8 should have a similar effect and facilitate indolediazine formation, whilst it might be predicted that carboline derivatives should be the main products when the methoxyl is in position 5 or 7 .

11-Methoxy-3-keto-4-methyl-3 : 4-dihydro-4-carboline (XI) yields on reduction with sodium in boiling $n$-butyl alcoholic solution a base, $\mathrm{C}_{13} \mathrm{H}_{16} \mathrm{ON}_{2}$, which, since it may be obtained by applying the same reduction process to norharmine methosulphate (XIII), must be regarded as $N$-methyltetrahydronorharmine (XIV). The identity of the substance prepared by these two methods is a satisfactory proof of the position of the methoxyl group in the molecules of the bases of Peganum harmala. 


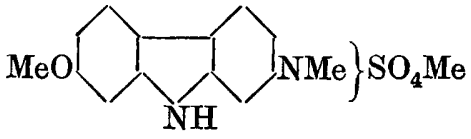

(XIII.*)

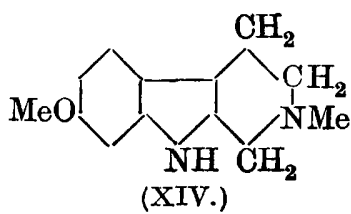

(XIV.)

\section{The Constitution of Harmaline.}

In Part III of this research there was described a neutral substance, $\mathrm{C}_{13} \mathrm{H}_{14} \mathrm{O}_{2} \mathrm{~N}_{2}$, m. p. $228^{\circ}$, which results from the oxidation of harmaline methosulphate by means of potassium permanganate in acetone solution, and it was pointed out that, if harmaline has the constitution $X V$, this substance will be represented by the expression XVI, whilst if the alternative formula XVII be taken for harmaline, the substance, m. p. $228^{\circ}$, must be represented by formula XVIII.

(XV.)

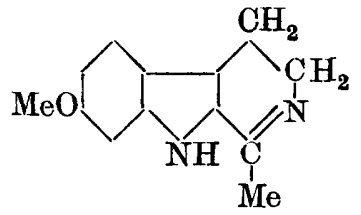

(XVII.)

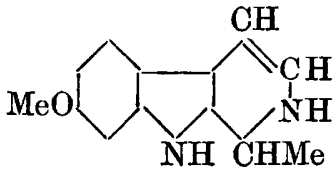

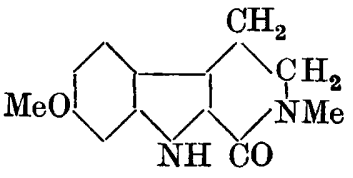

(XVI.)

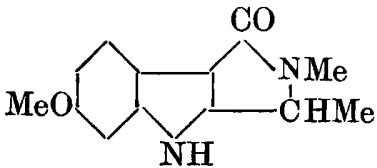

(XVIII.)

Since, however, the substance, m. p. $228^{\circ}$, yields, on reduction with sodium and $n$-butyl alcohol, $N$-methyltetrahydronorharmine identical with the base obtained as described above, there is no longer any ambiguity, and the oxidation product of harmaline methosulphate must have the constitution symbolised in XVI. Consequently harmaline is represented by the expression XV. Synthetically prepared $N$-methyltetrahydronorharmine has been oxidised by potassium permanganate in acetone solution with

* The bracket employed in this formula and in similar expressions indicates the salt-like constitution of the substance which is thus symbolised in harmony with Werner's conception of the nature of ammonium and other onium complexes. It is now recognised on all hands that the ionisable bonds (electro. valencies) in salts differ so much, not perhaps in kind but in degree, from the valencies (co-valencies) which occur in typical carbon compounds as to demand a distinctive notation. It may be recalled that the experimental proof of Werner's theory has been furnished by Meisenheimer (Annalen, 1913, 397, 273). The plan of using the bracket is not only theoretically sound, but also convenient, as it would be an embarrassment to be forced to decide to which of the nitrogen atoms the chlorine is attached in such substances as methylpropylharmine chloride (formulæ XXII and XXIII). 
formation of the substance melting at $228^{\circ}$, and this observation is of value inasmuch as it renders possible the proof of identity of a synthetic compound with one prepared from harmaline.

\section{The Constitution of Alkylated Harmine Derivatives.}

It has already been suggested (Part III, p. 942) that the saltforming nitrogen atom in the harmine derivatives is that which is situated in the pyridine nucleus, and the formula XIX was assigned to methylharmine. On a similar assumption, norharman methosulphate would be represented by the expression $\mathrm{XX}$.

(XIX.) $\mathrm{MeO}$

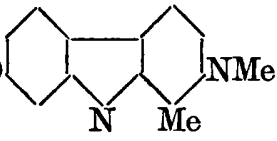

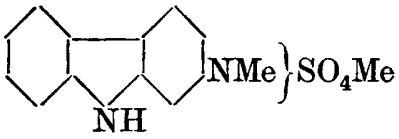

(XX.)

So far as the position of the methyl group is concerned, this hypothesis now receives definite experimental proof, since we have found that norharman methosulphate, on oxidation with potassium ferricyanide in alkaline solution, yields the ketomethyldihydrocarboline (VII), the synthesis of which is mentioned above. The further alkylation of methylharmine should, on the basis of our views, occur at the indole-nitrogen atom and dimethylharmine chloride should be constituted as shown in formula XXI. In order to obtain evidence in support of this theory, we have prepared isomeric methylpropylharmine chlorides by introducing the alkyl groups in a different order. The action of methyl iodide on propylharmine followed by conversion to the chloride by means of silver chloride yields the salt XXII, which closely resembles, but is not identical with, the isomeride (XXIII) prepared from methylharmine and propyl iodide followed by treatment of the product with silver chloride.
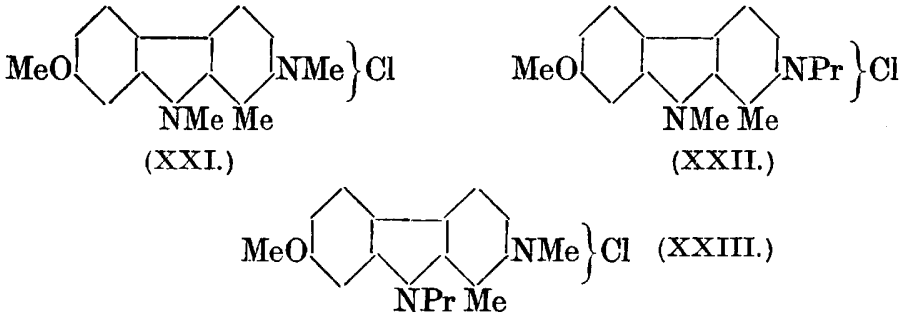

(XXIII.)

Before we were acquainted with the remarkable effect of the substitution of methyl for hydrogen in the - $\mathrm{CO}-\mathrm{NH}-$ group of indole-2-carboxyacetalylamides on the direction of closing the ring, it seemed that dimethylharmine chloride was a very suitable object of a synthetical investigation, and a plan of attack was 
formulated in Part V (p. 1613). The necessary starting point, namely, 6-methoxy-1-methylindole-2-carboxylic acid (XXVI), has been prepared, but, in view of the interesting developments set out above which have enabled our goal to be reached in another way, the further stages of the synthesis have not yet been attempted. $m$-Anisidine was methylated by means of methyl iodide, and the crude methyl- $m$-anisidine directly converted into a nitroso-derivative, which was reduced to $m$-methoxyphenylmethylhydrazine (XXIV) by means of zinc dust in glacial acetic acid solution below $10^{\circ}$. This was readily converted into pyruvic acid $m$-methoxyphenylmethylhydrazone $(X X V)$, but no little difficulty was experienced in inducing the indole transformation until it was found that the reaction proceeded smoothly when the hydrazine was dissolved in saturated alcoholic hydrochloric acid.

$\mathrm{MeO} / \mathrm{NMe} \cdot \mathrm{NH}_{2}$

(XXIV.)

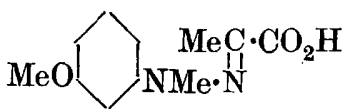

(XXV.)

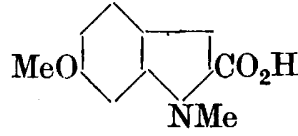

(XXVI.)

That the indole ring is closed in the $p$-position to the methoxyl group is rendered very probable by a consideration of the behaviour of hydrazones derived from $m$-methoxyphenylhydrazine, which we have already proved yield 6-methoxyindole derivatives (Part V, p. 1614).

We have already shown (Part V, p. 1617) that harman (III) may be obtained from tryptophan by condensation with acetaldehyde followed by oxidation of the product, and it is obvious that if methoxytryptophan (XXVII) could be obtained it should be converted into harmine in a similar manner. Some progress has been made in this direction and the indications that this synthesis is possible are unmistakable (see p. 1s84), but the experimental work involved is very tedious and consumes so much time, labour, and expensive material that the investigation has not yet been brought to a conclusion. The starting point was 6 -methoxyindole (XXVIII), which was prepared from 6-methoxyindole-2-carboxylic acid (XXIX) by a modification of the method already described (Part V,

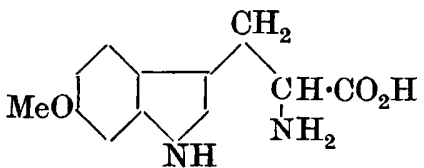

(XXVII.)

(XXX.)<smiles>COCCC1CCCN1</smiles>

(XXVIII.)<smiles>COCC1COC(=O)N1</smiles>

(XXIX.)<smiles>COCC1C2CCNCC1CC2</smiles>

(XXXI.) 
HARMINE AND HARMALINE. PART VI ${ }_{\text {View Article Online }}$
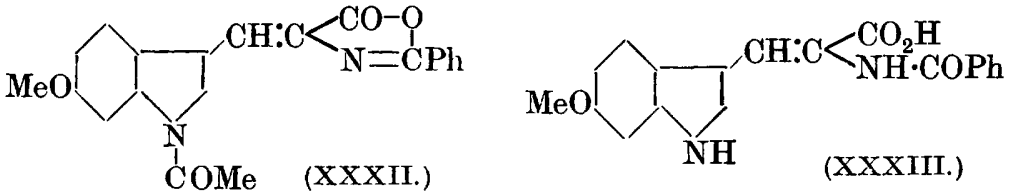

p. 1632). 6-Methoxyindole was transformed into 6-methoxyindole3-aldehyde (XXX) and 3-chloro-7-methoxyquinoline (XXXI) by the action of chloroform and potassium hydroxide in aqueous alcoholic solution. This interesting aldehyde was then condensed with hippuric acid in presence of dry sodium acetate and acetic anhydride, and the lactone (XXXII) thus produced at once hydrolysed by means of aqueous sodium hydroxide with formation of $\alpha$-benzoylamino- $\beta$-[6-methoxyindyl(3)]-acrylic acid (XXXIII). On reduction, hydrolysis, condensation with acetaldehyde, and oxidation, a minute amount of a base which is probably harmine was isolated, but the quantity obtained was insufficient to establish this important result with certainty (p. 1884). An account of the behaviour of some of the substances now described on treatment with magnesium methyl iodide is reserved for a future communication. It may be mentioned here, however, that 3-chloro-1-methyl4-carboline (XXXIV), which may be prepared under closely defined conditions from 3-keto-1-methyl-3:4-dihydro-4-carboline (V) by the action of phosphorus pentachloride and phosphoryl chloride, does not appear to react with the Grignard reagent.

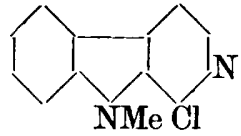

(XXXIV.)

EX P E I M E T A L.

\section{6-Methoxyindole (Formula XXVIII).}

It was mentioned in Part $V$ of this research (p. 1632) that some 6-methoxyindole had been obtained by the action of heat on 6 methoxyindole-2-carboxylic acid, but the yield was small and we have since found that the following modification, which consists in decomposing the ammonium salt in the place of the free acid, gives much better results. The dry ammonium salt of the acid (loc. cit., p. 1632), in quantities of 5 grams, contained in a boiling-tube fitted with a spiral glass condenser to the end of which a test-tube is attached, is gradually heated with a small flame. The salt melts and effervesces and when decomposition is complete any sublimate of methoxyindole and ammonium carbonate is washed into the boiling tube with a little hot water, alcohol is added, and the whole heated to boiling, when all dissolves except a small quantity of a 
black tar. The liquid is decanted and, on cooling, deposits crude 6-methoxyindole (88 per cent. of theory), which is collected, dried in a vacuum desiccator, and crystallised from light petroleum, from which it separates in plates melting at $91-92^{\circ}$. The yield of pure substance is about 55 per cent. of that theoretically possible.

The picrate separated in red needles when solutions of the indole and picric acid in benzene were mixed and was purified for analysis by recrystallisation from benzene containing a little light petroleum, from which the derivative separated in brilliant red needles melting at $137^{\circ}$ [Found: $\mathrm{N}=15^{\circ} \cdot \mathrm{C}_{9} \mathrm{H}_{9} \mathrm{ON}, \mathrm{C}_{6} \mathrm{H}_{2}(\mathrm{OH})\left(\mathrm{NO}_{2}\right)_{3}$ requires $\mathrm{N}=14.9$ per cent.].

\section{6-Methoxy-1-methylindole-2-carboxylic Acid (Formula XXVI).}

The interest attaching to this acid is discussed in the introduction to the present communication (p. 1877; compare also Part V, p. 1613) and the method of synthesis is also sketched. The experimental details of the various stages in the synthesis are as follows :

I. Preparation of Methyl-m-anisidine, $\quad \mathrm{MeO}^{\circ} \mathrm{C}_{6} \mathrm{H}_{4} \cdot \mathrm{NHMe}$-- In preparing this substance, $m$-anisidine $(30.8$ grams), contained in a flask connected with a reflux condenser, was mixed in the cold with methyl iodide (39 grams), when the temperature gradually rose and in a short time a vigorous reaction set in. When this had subsided, the semi-solid mass was heated on the steam-bath for several hours, mixed with water and dilute sodium hydroxide, and extracted with ether. The ethereal solution, dried over potassium carbonate, was evaporated and the residue fractionated under reduced pressure, when $32 \cdot 3$ grams distilled at $133-135^{\circ} / 12 \mathrm{~mm}$. as a colourless liquid which doubtless consisted of methyl-m-anisidine mixed with unchanged $m$-anisidine and some of the dimethyl derivative.

This oil ( $7 \cdot 2$ grams) was added to a mixture of ice ( 20 grams) and concentrated hydrochloric acid ( 8 c.c.), and sodium nitrite ( 4 grams) dissolved in water (12 c.c.) run in drop by drop during half an hour, the whole being well cooled all the time. The product, which should just react with starch-potassium iodide paper, was extracted three times with ether, the ethereal solution dried over calcium chloride and evaporated in a weighed flask, when 4 grams of crude nitrosomethyl-m-anisidine remained as a dark syrup.

II. m-Methoxyphenylmethylhydrazine, $\quad \mathrm{MeO} \cdot \mathrm{C}_{6} \mathrm{H}_{4} \cdot \mathrm{NMe} \cdot \mathrm{NH}_{2} \cdot-$ This substance was prepared by slowly adding the solution of crude nitrosomethyl-m-anisidine ( 4 grams) in glacial acetic acid $(8$ c.c.) to a well-stirred mixture of zinc dust ( 16 grams) and water (28 c.c.), the temperature being kept below $10^{\circ}$. The product was kept over-night, again well stirred, and heated on the steam-bath for a few minutes, filtered, the filtrate made alkaline with sodium 
hydroxide and extracted several times with ether. This extract, as well as the washings of the zinc dust with ether, was dried over potassium carbonate and the ether distilled off, when crude $m$ methoxyphenylmethylhydrazine (3 grams) remained as a pale brown syrup and was sufficiently pure for the next step.

III. Pyruvic Acid m-Methoxyphenylmethylhydrazone (Formula XXV) and (6 ?)-Methoxy-1-methylindole-2-carboxylic Acid (Formula XXVI).-When crude $m$-methoxyphenylmethylhydrazine (3 grams) was dissolved in acetic acid ( 30 c.c. of 15 per cent.) and then pyruvic acid (4 grams) added, a yellow oil separated, which, on standing and rubbing, solidified. After remaining over-night, the crystalline mass was collected, dried in a vacuum, and crystallised from light petroleum, from which pyruvic acid $\mathrm{m}$-methoxyphenylmethylhydrazone separated in lemon-yellow needles melting at $66^{\circ}$ (Found : $\mathrm{C}=59 \cdot 7$; $\mathrm{H}=6.5 . \quad \mathrm{C}_{11} \mathrm{H}_{14} \mathrm{O}_{3} \mathrm{~N}_{2}$ requires $\mathrm{C}=59.5 ; \mathrm{H}=6.3$ per cent.). This hydrazone dissolves in sodium carbonate to a deep orange solution and on acidifying with hydrochloric acid, the colour changes to yellow and then rapidly fades owing no doubt to hydrolysis.

After a number of experiments on the action of aqueous hydrochloric acid under different conditions on this hydrazone, during which varying quantities of (6 ?)-methoxy-1-methylindole-2carboxylic acid, mixed generally with a red amorphous substance completely soluble in glacial acetic acid, were isolated, the following satisfactory process for the preparation of this acid was developed. The $m$-methoxyphenylmethylhydrazone of pyruvic acid was added to about ten times its weight of a saturated alcoholic solution of hydrogen chloride, when it quickly dissolved to a pale red solution and in a minute or two, crystals commenced to separate and the temperature rose considerably. After an hour, the crystals were collected, washed with alcohol, then with water, again with alcohol, and dried in a vacuum desiccator. The acid is sparingly soluble in alcohol and separates in small, stout needles with pointed ends (Found : $\mathrm{C}=64 \cdot 5 ; \mathrm{H}=5 \cdot 5 . \quad \mathrm{C}_{11} \mathrm{H}_{11} \mathrm{O}_{3} \mathrm{~N}$ requires $\mathrm{C}=64 \cdot 4$; $\mathbf{H}=\mathbf{5 \cdot 4}$ per cent.). (6 ?)-Methoxy-1-methylindole-2-carboxylic acid decomposes at about $235^{\circ}$ with evolution of gas. It gives, in alcoholic solution with $p$-dimethylaminobenzaldehyde and hydrochloric acid, a purple solution which fades on boiling. A small quantity of the acid was cautiously heated until decomposition was complete, and the residue dissolved in boiling water; the filtered solution deposited, on cooling, an oil which is evidently the corresponding methoxymethylindole. In aqueous solution this substance gives with $p$-dimethylaminobenzaldehyde and hydrochloric acid (Ehrlich's reagent) * a red coloration which becomes intense on the addition

* This reagent was made by adding concentrated hydrochloric acid (20 c.c.) to the solution of $p$-dimethylaminobenzaldehyde (1 gram) in alcohol (119 e.c.). 
View Article Online

of sodium nitrite and shows a strong absorption band in the yellow end of the green. With a pine shaving, a purple reaction is obtained. The addition of a solution of picric acid in benzene to the solution of methoxymethylindole in the same solvent, causes a well-defined picrate to separate in red-brown needles which melt at $123^{\circ}$.

\section{6-Methoxyindole-3-aldehyde (Formula XXX) and 3-Chloro- 7-methoxyquinoline (Formula XXXI).}

These substances were obtained from 6-methoxyindole by treatment with chloroform and potassium hydroxide substantially under the conditions recommended by Ellinger (Ber., 1906, 39, 2520) for the preparation of indole-3-aldehyde and 3-chloroquinoline from indole. In a flask attached to a reflux condenser and fitted with a stirrer and dropping funnel, 6-methoxyindole (10 grams) is mixed with alcohol (100 c.c. of 96 per cent.), water (20 c.c.), and chloroform (36 c.c.), and the whole heated on the steam-bath just to boiling. Potassium hydroxide (25 grams), dissolved in water (25 c.c.) and made up to 250 c.c. with alcohol (96 per cent.), is dropped in continuously during two and a half hours, the whole being very vigorously stirred. The product is boiled for half an hour, the alcohol and chloroform are distilled off, and the residue is distilled in steam until the distillate gives hardly any coloration on the addition of dilute nitric acid and sodium nitrite. The hot liquid in the flask is quickly filtered and allowed to cool over-night, when 6-methoxyindole-3-aldehyde (0.8 gram) separates in brown needles and, after recrystallisation from water with the aid of animal charcoal, the aldehyde is obtained in almost colourless needles (Found : $\mathrm{C}=68 \cdot 3$; $\mathrm{H}=5 \cdot 1 ; \quad \mathrm{N}=8 \cdot 0 . \quad \mathrm{C}_{10} \mathrm{H}_{9} \mathrm{ON} \quad$ requires $\mathrm{C}=68.5 ; \quad \mathrm{H}=5 \cdot 1 ;$ $\mathrm{N}=8.0$ per cent.). 6-Methoxyindole-3-aldehyde melts at $185^{\circ}$ and gives with sulphuric acid a red coloration due probably to the formation of a dyestuff similar to that observed by Ellinger to be formed under the same conditions from indole-3-aldehyde.

3-Chloro-7-methoxyquinoline.-This interesting substance passes over along with unchanged methoxyindole when the product of the action of chloroform and potassium hydroxide on the latter is distilled in steam as described above. The distillate, which may amount to several litres, is extracted four times with ether, the ethereal solution thoroughly shaken with several small quantities of dilute hydrochloric acid (20 per cent.), dried, and evaporated, when 6-methoxyindole (about 7 grams) remains in an almost pure condition. The acid extract is made alkaline with sodium hydroxide, and air passed to remove the dissolved ether, when needles of the chloromethoxyquinoline soon commence to separate. After some hours, the needles (1 gram) are collected, washed, and recrystallised 
HARMINE AND HARMALINE. PART VI View Article Online

from a large amount of boiling water (Found : $\mathrm{N}=7 \cdot 5 ; \mathrm{Cl}=18 \cdot 1$. $\mathrm{C}_{10} \mathrm{H}_{8} \mathrm{ONCl}$ requires $\mathrm{N}=7 \cdot 2 ; \mathrm{Cl}=18 \cdot 3$ per cent.).

3-Chloro-7-methoxyquinoline melts at $91^{\circ}$, has a curious odour somewhat resembling that of anethole, and is readily soluble in dilute acids. The solution in dilute sulphuric acid, but not in hydrochloric acid, has a strong blue fluorescence, and the addition of hydrochloric acid to the sulphuric acid solution destroys this fluorescence.

The chlorine atom is very firmly fixed, since, after heating with concentrated aqueous ammonia in a sealed tube at $100^{\circ}$ for five hours, the substance is recovered unchanged.

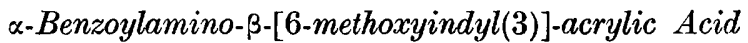

(Formula XXXIII).

The anhydride of this substance is obtained when 6-methoxyindole-3-aldehyde is condensed with hippuric acid (compare Ellinger and Flamand, Ber., 1907, 40, 3031). The aldehyde (1 gram), hippuric acid (1.4 grams), and freshly fused sodium acetate $(0.55$ gram) are finely ground, mixed with freshly distilled acetic anhydride $(2.5$ c.c.), and heated in a test-tube in a boiling-water bath for fifteen minutes with occasional stirring, care being taken to exclude moisture. The mixture becomes liquid and dark red and, after cooling, the viscous mass is rubbed with warm water and then boiled with water ( 200 c.c.) and filtered hot; the brittle residue is again ground and extracted with boiling water (100 c.c.). The residual crude az-lactone was not recrystallised, but at once hydrolused by boiling the finely divided substance with sodium hydroxide (100 c.c. of 1 per cent.) until the odour of ammonia could not be detected. After the addition of dilute hydrochloric acid until the reaction was just acid to Congo-paper, and standing over-night, the precipitate was collected and recrystallised from dilute alcohol, from which it separated in brown-yellow needles. Analysis showed that this substance is benzoylamino(methoxyindyl)acrylic acid (Found: $\mathrm{C}=67 \cdot 3 ; \quad \mathrm{H}=4 \cdot 8 . \quad \mathrm{C}_{19} \mathrm{H}_{16} \mathrm{O}_{4} \mathrm{~N}_{2}$ requires $\mathrm{C}=67 \cdot 8$; $\mathrm{H}=4.8$ per cent.). This substance softens at $215^{\circ}$, melts with decomposition at $230^{\circ}$, and is insoluble in water, but readily so in alcohol; it dissolves in acetic anhydride, and on boiling a red colour develops presumably due to regeneration of the az-lactone.

This acid was reduced in aqueous solution by means of a large excess of sodium amalgam, and the strongly alkaline solution then boiled for ten minutes. The liquid was acidified with dilute sulphuric acid, and a little acetaldehyde added while still hot. This resulted in the formation of a cloud, and condensation obviously occurred. Excess of 5 per cent. potassium dichromate was then 
added and the solution boiled and rendered strongly alkaline. The base, if any, was extracted by means of ethyl acetate, and the separated ethyl acetate solution washed with dilute hydrochloric acid. This solution exhibited a blue fluorescence, but nothing could be isolated from it. A more definite result was obtained by reducing the acid in acetic acid solution by shaking with hydrogen after the addition of palladious chloride and gum arabic. After boiling and cooling, the solution was diluted, filtered, and evaporated to dryness in a vacuum. Alcoholic barium hydroxide was added and the solution boiled during an hour, then diluted, rendered just acid with sulphuric acid, and two drops of acetaldehyde added. After heating on the steam-bath for about thirty seconds, a large excess of 5 per cent. potassium dichromate was added and the solution boiled. Sodium hydroxide in excess was then added and the mixture extracted with much ethyl acetate. The filtered extract was shaken with dilute hydrochloric acid, and the strongly fluorescent aqueous solution evaporated to a small bulk. On rendering alkaline, there was a small precipitate, and this was collected and dissolved in methyl alcohol. The solution was concentrated and deposited, on cooling, a minute amount of needle crystals resembling harmine in appearance. These melted at $220-230^{\circ}$ and were obviously impure, but mixed with harmine, the melting point was raised to $225-245^{\circ}$. Pure harmine melts at $257^{\circ}$, and it is unfortunate that the amount of base obtained in this experiment did not admit of repeated crystallisation. We have no doubt that this substance consisted of impure harmine, and the fluorescence in acid solution was identical with that exhibited by harmine salts.

\section{3-Chloro-1-methyl-4-carboline (Formula XXXIV).}

This substance is obtained when 3-keto-1-methyl-3:4-dihydro4-carboline (m. p. $242^{\circ}$; compare Part V, p. 1638) is treated with phosphorus oxychloride and pentachloride. Unless special conditions are observed, the yield is poor, and experience has shown that the essential points to be observed are that the phosphorus pentachloride is pure and dry, that the oxychloride is distilled immediately before use, and the carboline should be as pure as possible. It is also important that excess of pentachloride should be avoided. The carboline $(0.5 \mathrm{gram}) *$ is mixed in a tube with the oxychloride (6 grams) and then the pentachloride ( 1 gram)

* A peculiar characteristic of this substance may be mentioned. It is almost insoluble in water and very sparingly soluble in absolute alcohol, but it dissolves much more readily in alcohol containing water. It separates from water or alcohol in long needles, but from a mixture of alcohol and water in glistening plates. 
added. The whole is well stirred, the tube sealed, and heated in the boiling-water bath for six hours. The contents of the tube are poured on to ice and after the oxychloride has been completely decomposed, the solid is collected, washed with dilute ammonia, dried, and recrystallised from light petroleum (b. p. 80-100 ${ }^{\circ}$ ) containing some benzene, from which the substance separates in colourless needles (Found: $\mathrm{C}=66.2 ; \quad \mathrm{H}=4 \cdot 1 . \quad \mathrm{C}_{12} \mathrm{H}_{9} \mathrm{~N}_{2} \mathrm{Cl}$ requires $\mathrm{C}=66.6 ; \mathrm{H}=4 \cdot 1$ per cent.).

3-Chloro-1-methyl-4-carboline melts at $110^{\circ}$, and is feebly basic, since it yields with concentrated hydrochloric acid a rather sparingly soluble, yellow hydrochloride, which is dissociated by water. The acid solutions do not fluoresce. When the solution in benzene is mixed with a solution of picric acid in the same solvent, a yellow, crystalline picrate separates which melts at $213^{\circ}$. Attempts were made to convert this substance into methyl harman by the action of magnesium methyl iodide in boiling isoamyl ether solution, but the only product isolated was keto-1-methyldihydro-4-carboline (m. p. $242^{\circ}$ ). There is no doubt that a little methylharman is produced, as the acid washings of the isoamyl ethereal solution, obtained on adding water to the reaction mixture, exhibited violet fluorescence. The actual amount of the base must, however, be very small.

Action of Methylamine on Bromodimethylacetal. Formation of Methylaminodimethylacetal, $\mathrm{NHMe} \cdot \mathrm{CH}_{2} \cdot \mathrm{CH}(\mathrm{OMe})_{2}$, and Methylaminobisdimethylacetal, $\mathrm{NMe}\left[\mathrm{CH}_{2} \cdot \mathrm{CH}(\mathrm{OMe})_{2}\right]_{2}$.

The researches of Freundler and Ledru (Compt. rend., 1905, 140, 794 ; Bull. Soc. chim., 1907, [iv], 1, 71) on the action of alcohols on the product of the bromination of paraldehyde have rendered the bromoacetals, and indirectly the aminoacetals, readily accessible substances. Thus, for example, methylaminodimethylacetal results from the action of methylamine on bromodimethylacetal, and as this substance does not appear to have been described and we required it in considerable quantity for the present investigation, we have worked out the following method for its preparation. Bromodimethylacetal (150 grams) is mixed with methyl alcohol (400 c.c.) in which the methylamine obtained by heating 500 grams of the pure hydrochloride with concentrated aqueous potassium hydroxide has been absorbed, and the whole heated in an autoclave at $140^{\circ}$ for twelve hours. The methyl alcohol and excess of methylamine are distilled off under $200 \mathrm{~mm}$. pressure and used in a subsequent preparation, an equal volume of water is added to the syrupy residue, and the solution, which contains no unchanged bromodimethylacetal, is mixed with a large excess of anhydrous potassium 
carbonate and extracted four times with much ether. The ethereal solution, thoroughly dried over potassium carbonate, is evaporated and the residue $(85$ grams) quickly distilled under $10 \mathrm{~mm}$. pressure, all below $100^{\circ}$ being collected (the residue [A] is described below).

On fractionating the distillate at the ordinary pressure, methylaminodimethylacetal is readily obtained pure as a limpid oil which has an odour resembling, but more pungent than, that of aminoacetal, and distils at $140^{\circ} / 760 \mathrm{~mm}$. (Found : $\mathrm{N}=11.9 . \quad \mathrm{C}_{5} \mathrm{H}_{13} \mathrm{O}_{2} \mathrm{~N}$ requires $\mathrm{N}=11.8$ per cent.).

The fraction of higher b. p. (A) was collected from several experiments and fractionated under reduced pressure, when the main portion distilled at $100-105^{\circ} / 10 \mathrm{~mm}$. as a rather viscid, colourless oil and evidently consisted of methylaminobisdimethylacetal (Found : $\mathrm{N}=6.9 . \quad \mathrm{C}_{9} \mathrm{H}_{21} \mathrm{O}_{4} \mathrm{~N}$ requires $\mathrm{N}=6.7$ per cent.).

Aminodimethylacetal, $\mathrm{NH}_{2} \cdot \mathrm{CH}_{2} \cdot \mathrm{CH}(\mathrm{OMe})_{2}$ (b. p. $135^{\circ} / 755 \mathrm{~mm}$.) and aminobisdimethylacetal, $\mathrm{NH}\left[\mathrm{CH}_{2} \cdot \mathrm{CH}(\mathrm{OMe})_{2}\right]_{2}$ (b. p. $163^{\circ}$ ) $84 \mathrm{~mm}$.) are readily obtained by heating bromodimethylacetal (150 grams) in an autoclave with alcohol (500 c.c.) which has been saturated with ammonia at $0^{\circ}$ for ten hours. In working up the product, care must be taken to saturate thoroughly the residue, after distilling off the alcohol and ammonia, with potassium carbonate and to extract several times with much ether, otherwise much of the aminodimethylacetal may be lost. As these aminodimethylacetals are somewhat volatile in ether vapour, the ether used for extractions should be employed in subsequent preparations.

\section{3-Keto-4-methyl-3 : 4-dihydro-4-carboline (Formula VII).*}

In the preparation of this substance from indole-2-carboxylic acid, the chloride of the acid was first made by the action of phosphorus pentachlorine on the suspension of the acid in acetyl chloride, and the solution of the acid chloride in chloroform then treated with methylaminodimethylacetal, $\mathrm{NHMe} \cdot \mathrm{CH}_{2} \cdot \mathrm{CH}(\mathrm{OMe})_{2}$ (compare Part V, p. 1626). After the chloroform had been removed by evaporation, water was added, when an oil separated which soon crystallised. The solid was collected, dried on porous porcelain in a vacuum desiccator, and recrystallised twice from a mixture of benzene and light petroleum, from which colourless needles of indole-2-carboxydimethylacetalylmethylamide separated; these melted at $104^{\circ}$ (Found: $\mathrm{C}=64 \cdot 3 ; \mathrm{H}=7 \cdot 0 ; \mathrm{N}=10 \cdot 6$. $\quad \mathrm{C}_{14} \mathrm{H}_{18} \mathrm{O}_{3} \mathrm{~N}_{2}$ requires $\mathrm{C}=64.1 ; \mathrm{H}=6.9 ; \mathrm{N}=10.7$ per cent.). In order to close the ring, this substance was added to a saturated alcoholic

* In strict accordance with the nomenclature suggested in Part IV (p. 970) for carboline derivatives, the substance described in Part V (p. 1638) as 2-keto-1-methyl-2 : 3-dihydro-4-carboline should have been named 3-keto1-methyl-3 : 4-dihydro-4-carboline. 
solution of hydrogen chloride at $40^{\circ}$, when a clear solution resulted, from which, especially if seeded, crystals of the hydrochloride of ketomethyldihydrocarboline soon began to separate. On the addition of water, a white precipitate was produced, which was collected, washed with water, then with dilute ammonia, and dried; in this condition the substance melts at about $240-255^{\circ}$ and yields a green colour with Ehrlich's reagent (p. 1881) which deepens on heating and becomes almost colourless on cooling. After twice crystallising from alcohol, the long, white needles melt at $263^{\circ}$ and no longer give this reaction (Found : $\mathrm{C}=64 \cdot 3 ; \mathrm{H}=7 \cdot 0 ; \mathrm{N}=10 \cdot 6$. $\mathrm{C}_{14} \mathrm{H}_{18} \mathrm{O}_{3} \mathrm{~N}_{2}$ requires $\mathrm{C}=64.1 ; \mathrm{H}=6.9 ; \mathrm{N}=10.7$ per cent.).

3-Keto-4-methyl-3:4-dihydro-4-carboline combines with hydrochloric acid to yield a colourless hydrochloride, the solution of which exhibits a faint though perfectly distinct fluorescence. A trace of the substance, heated with zinc dust in a narrow test-tube, gives a sublimate, the solution of which in dilute hydrochloric acid exhibits a marked blue fluorescence. These properties indicate that ring formation in the case of indole-2-carboxydimethylacetalylmethylamide has resulted in the formation of the carboline and not of 5-keto-4 : 5-dihydroindolediazine $(1: 4)$ (compare p. 1874, and also Part V, p. 1627). It is of considerable interest (see p. 1877) that this substance may be prepared from norharman (Part V, p. 1619) in the following manner. Norharman $(0.5 \mathrm{gram})$, dissolved in boiling benzene (10 c.c.), was converted into its methosulphate by the addition of dry methyl sulphate $(1$ c.c.) by boiling the solution during an hour. The derivative separated in a crystalline condition and the benzene was decanted. The residue was then dissolved in water (50 c.c.), rendered just alkaline by the addition of potassium hydroxide and heated on the steam-bath. A solution of potassium ferricyanide was then cautiously added until ferricyanide remained in excess. A colourless substance separated in needles and, after cooling, this was collected and crystallised from alcohol. It was obtained in slender needles melting at $263^{\circ}$ alone or mixed with synthetical 3-keto-4-methyl-3:4-dihydro-4-carboline obtained as described above.

On distillation over a large excess of zinc dust in a stream of hydrogen, a crystalline distillate was obtained. This consisted chiefly of indole, but was found to contain a little norharman. The product was extracted with dilute acid and the picrate prepared. After several crystallisations, a minute amount of material, crystallising from hot water in woolly yellow needles, decomposing at $260^{\circ}$, was obtained. The substance thus appeared to be identical with norharman picrate, and it should also be noted that the acid solution of the base exhibited intense blue fluorescence. 
3-Methylindole-2-carboxydimethylacetalylmethylamide and 5-Keto4 : 7-dimethyl-4 : 5-dihydroindolediazine $(1: 4)$,
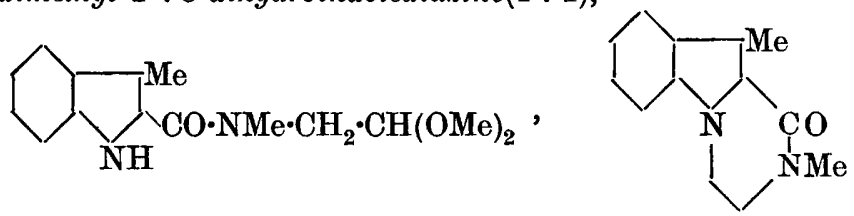

3-Methylindole-2-carboxylic acid (scatole-2-carboxylic acid (see Part V, p. 1634) was converted into the acid chloride by means of phosphorus pentachloride and acetyl chloride, and this, dissolved in chloroform, treated with methylaminodimethylacetal (compare Part V, p. 1626). On adding water, an oil separated which, especially when vigorously rubbed, gradually solidified. This crude 3-methylindole-2-carboxydimethylacetalylmethylamide was collected, dried on porous porcelain, and crystallised first from a mixture of benzene and light petroleum and then from light petroleum alone, from which it separated in small, flat plates which melt at $87-88^{\circ}$ and are readily soluble in most of the usual organic solvents (Found: $\mathrm{C}=65.2 ; \quad \mathrm{H}=7.2 ; \mathrm{N}=10.0 . \quad \mathrm{C}_{15} \mathrm{H}_{30} \mathrm{O}_{3} \mathrm{~N}_{2}$ requires $\mathrm{C}=65 \cdot 2 ; \mathrm{H}=7 \cdot 2 ; \mathrm{N}=10 \cdot 1$ per cent.).

When this substance was added to a saturated alcoholic solution of hydrogen chloride at $40^{\circ}$, it dissolved and yellow crystals soon commenced to separate which, after some hours, were collected and recrystallised first from alcohol containing a little ammonia and then from alcohol alone, from which 5-keto-4:7-dimethyl-4:5dihydroindolediazine $(1: 4)$ separated in long, flat needles melting at $159^{\circ}$ (Found: $\mathrm{C}=73.6 ; \mathrm{H}=5 \cdot 7 ; \mathrm{N}=13.2 . \quad \mathrm{C}_{13} \mathrm{H}_{12} \mathrm{ON}_{2}$ requires $\mathrm{C}=73.7 ; \mathrm{H}=5 \cdot 7 ; \mathrm{N}=13.2$ per cent.).

In appearance and properties, this substance closely resembles 7-methyl-4 : 5-dihydroindolediazine(1:4) (see Part V, p. 1635). Concentrated hydrochloric acid converts it into a yellow crystalline hydrochloride, which is somewhat soluble in hydrochloric acid to a yellow solution exhibiting no fluorescence, although the neutral or alkaline solutions of the base have a slight blue fluorescence.

On distillation with zinc dust, it develops an odour resembling that of indole and yields a yellow base which has not been further investigated; in this respect it behaves very much like 5-keto-4 : 5 dihydroindolediazine $(1: 4)$, which in these circumstances yields indole and an orange base (Part V, p. 1628). On boiling with Ehrlich's reagent (p. 1881), a bluish-green coloration is obtained which almost disappears on cooling, and on warming with vanillin and concentrated hydrochloric acid, a deep purple colour rapidly develops and remains on dilution with water, but is almost dissharged on rendering alkaline. 
6-Methoxyindole-2-carboxydimethylacetalylmethylamide (Formula X), 11-Methoxy-3-keto-4-methyl-3:4-dihydro-4-carboline (Formula $\mathrm{XI})$, and 11-Methoxy-5-keto-4-methyl-4:5-dihydroindolediazine(1 : 4) (Formula XII).

This series of substances, which represent the closing simultaneously of the carboline and indolediazine rings (p. 1875), were obtained under the following conditions.

6-Methoxyindole-2-carboxylic acid was first converted into the acid chloride, and this combined with methylaminodimethylacetal in chloroform solution in the usual manner (compare Part V, p. 1626).

After distilling off the chloroform and adding water to the residue, 6-methoxyindole-2-carboxydimethylacetalylmethylamide separated at once in good yield and in an almost pure condition, since the melting point was $127^{\circ}$. It crystallised readily from light petroleum in colourless needles, the melting point rising to $129^{\circ}$ (Found : $\mathrm{C}=61.6$; $\mathrm{H}=6.9 ; \quad \mathrm{N}=9.5 . \quad \mathrm{C}_{15} \mathrm{H}_{20} \mathrm{O}_{4} \mathrm{~N}_{2}$ requires $\mathrm{C}=61 \cdot 6 ; \quad \mathrm{H}=6.8 ;$ $\mathrm{N}=9 \cdot 6$ per cent.).

Action of Alcoholic Hydrogen Chloride.-When the methylamide just described is added to a saturated alcoholic solution of hydrogen chloride at $40^{\circ}$, it dissolves and crystallisation usually takes place very slowly, but after twenty-four hours a yellow crust will have separated. This is filtered off, ground with very dilute ammonia, and the colourless precipitate collected by the aid of the pump, dried, and crystallised from alcohol. In this condition the substance melted indefinitely at about $200^{\circ}$, and examination under the microscope confirmed the suspicion that it was a mixture, since it was seen to consist of slender needles as well as of rhombic plates. On distillation with zinc dust, a sublimate was obtained which fluoresced distinctly blue in acid solution and also gave a purple coloration when warmed with Ehrlich's reagent (p. 1881), a behaviour which suggested the presence both of a carboline and of an indolediazine. The separation of the two isomerides proved to be very difficult, but may be brought about by careful fractional crystallisation from alcohol repeated many times, the first crop consisting of the rhombic plates (m. p. $245-246^{\circ}$ ). It was then observed that the rhombic plates are more sparingly soluble in acetone than the needles. In our later experiments, the crude mixture was therefore first extracted with a little warm acetone, and the small insoluble portion repeatedly fractionally crystallised from acetone and from alcohol until no needles could be detected under the microscope.

11-Methoxy-3-keto-4-methyl-3:4-dihydro-4-carboline was thus obtained quite pure and melted at $245-246^{\circ}$ (Found : $\mathrm{C}=68.2$; $\mathrm{H}=5 \cdot 1 . \quad \mathrm{C}_{13} \mathrm{H}_{12} \mathrm{O}_{2} \mathrm{~N}_{2}$ requires $\mathrm{C}=68.4 ; \mathrm{H}=5.2$ per cent.). 
This carboline yields a colourless hydrochloride on mixing with hydrochloric acid and gives scarcely any coloration with Ehrlich's reagent (p. 1881). The solution of the hydrochloride exhibits a distinct though very slight blue fluorescence, but when a trace of the base is distilled with zinc dust, the sublimate yields, on dissolving in very dilute hydrochloric acid, a strongly fluorescent solution. This is possibly due to the production of norhamine.

The mother-liquors from the purification of the carboline contain the isomeric indolediazine and this may be isolated in a pure condition in the form of yellow needles melting at $205^{\circ}$ by repeated fractional crystallisation from alcohol (Found: $\mathrm{C}=68.70 ; \mathrm{H}=$ 5.24. $\mathrm{C}_{13} \mathrm{H}_{12} \mathrm{O}_{2} \mathrm{~N}_{2}$ requires $\mathrm{C}=68 \cdot 4 ; \mathrm{H}=5 \cdot 2$ per cent.).

11-Methoxy-5-keto-4-methyl-4:5-dihydroindolediazine $(1: 4)$ melts at $205^{\circ}$ and gives a greenish-blue colour on gently warming with Ehrlich's reagent. This becomes fainter and greener on cooling and the effects can be produced alternately. On adding hydrochloric acid, a bright yellowish-brown colour is developed; this becomes brown and greenish-brown on heating, returning to reddishbrown on cooling. The addition of sodium nitrite subsequent to the treatment with Ehrlich's reagent produces a dull green; little change is observed on heating and the solution remains bluish-green on dilution with water.

The indolediazine gives with vanillin and hydrochloric acid in the cold a deep red colour which becomes very intense on heating. After dilution with water, the solution is red by transmitted and blue by reflected light. The colour is destroyed by the addition of alkali. Neutral or alkaline, but not acid, solutions have a distinct blue fluorescence, and the yellow solution in acetone or benzene exhibits a very strong green fluorescence. The hydrochloride differs from the colourless hydrochloride of the isomeric carboline in being yellow.

\section{N-Methyltetrahydronorharmine (Formula XIV).}

11-Methoxy-3-keto-4-methyl-3 : 4-dihydro-4-carboline (1-3 grams) was dissolved in boiling $n$-butyl alcohol ( 20 c.c.), and sodium ( 2 grams) added. The solution became yellow and acquired a green fluorescence. In about two minutes ethyl alcohol was gradually added so as to keep the boiling solution clear and, when all the sodium had disappeared, dilute hydrochloric acid was added until an acid reaction to Congo was obtained. Steam was then passed into the brown-purple solution to remove the alcohols, and animal charcoal was added. The filtered solution was concentrated and, after cooling, rendered strongly alkaline by the addition of an excess of sodium hydroxide. This precipitated an ochre base which was 
collected and dissolved as far as possible in much boiling benzene. The solution was treated with animal charcoal, filtered, and concentrated by distillation. An orange-yellow substance separated in a crystalline form and this was recrystallised from toluene and then twice from methyl alcohol. It was thus obtained in colourless, prismatic needles melting at $182^{\circ}$, and at the same temperature when intimately mixed with a specimen prepared by the method next to be described. A careful comparison disclosed no difference existing between the two substances. This material was not analysed, as very little was available, but was dissolved in acetone, cooled to $-5^{\circ}$, and finely powdered potassium permanganate added in minute amounts until after some hours no further oxidation occurred. More acetone was added and the solvent boiled and filtered. On concentration and adding water, a small, crystalline precipitate was thrown down, and the substance was collected and twice crystallised from alcohol. The oxidation product was thus obtained in colourless, prismatic needles melting at $227^{\circ}$, and at the same temperature when mixed with an authentic specimen of keto- $N$-methyltetrahydronorharmine prepared by the oxidation of harmaline methosulphate (Part III, p. 959). N-Methyltetrahydronorharmine is best prepared from keto- $N$-methyltetrahydronorharmine by reduction with sodium in butyl alcoholic solution and except that the operation was conducted on a somewhat larger scale ( 5 grams of the substance) the details of the process are precisely similar to those given above. Indeed the phenomena noticed at all stages were so alike in the two cases as to afford ground for the belief that the first action of the reducing agent on the carboline derivative is to convert it into ketomethyltetrahydronorharmine. One of the most striking coincidences was that in each case the paper used for the filtration following the steam distillation developed, on standing in the air, a purple colour at the apex and a beautiful blueish-green nearer the edges. The substance prepared by this method also melted at $182^{\circ}$ (Found: $\mathrm{C}=72 \cdot 1 ; \quad \mathrm{H}=7 \cdot 3 . \quad \mathrm{C}_{13} \mathrm{H}_{16} \mathrm{ON}_{2}$ requires $\mathrm{C}=72 \cdot 2 ; \quad \mathrm{H}=7 \cdot 4$ per cent.). N-Methyltetrahydronorharmine is sparingly soluble in ether and very. sparingly soluble in benzene. It dissolves readily in methyl or ethyl alcohol. When freshly prepared and purified, it is colourless, but on standing in the air it quickly becomes brownish-yellow as the result of oxidation. In this respect it differs from $N$-methyltetrahydroharmine, which is much more stable. In order to show the relation of the substance to norharmine, the base has been prepared from norharmine methosulphate (Part III, p. 951) by reduction with sodium and butyl alcohol. The yield in this case was unsatisfactory. The metho- 
sulphate (1.8 grams) was dissolved in butyl alcohol (50 c.c.), and sodium (3 grams) added. The mixture was then boiled during twenty minutes by heating in an oil-bath, then diluted with alcohol, and the product isolated in the manner described above. The purification was difficult, but ultimately the substance was obtained pure and melted at $182^{\circ}$, and at the same temperature when mixed with a specimen made from ketomethyltetrahydronorharmine.

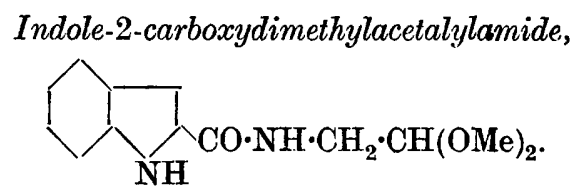

With the object of obtaining further evidence concerning the direction of ring formation in the case of the action of hydrogen chloride on dimethylacetalylamides, this comparatively simple case was investigated. The preparation of the acid chloride of indole-2-carboxylic acid and its conversion into indole-2-carboxyacetalylamide are described in detail in Part $V$ of this research (p. 1626), and in the present instance the same procedure was adopted except that aminodimethylacetal was employed in the place of aminoacetal and the condensation conducted in benzene instead of in chloroform solution, which appears to be an advantage. The acid chloride dissolves readily in boiling benzene and, after cooling and adding aminodimethylacetal ( $2 \mathrm{mols}$.), a viscid oil separates, which is not the case when chloroform is used as the solvent. The benzene was removed by distillation under reduced pressure, the residue mixed with water, and the solid product, obtained in a yield of 80 per cent. of theory dried in a vacuum desiccator. In this condition indole-2-carboxydimethylacetalylamide melts at $129^{\circ}$ and is nearly pure, but after crystallisation from benzene, from which it separates as a matted mass of colourless needles, the melting point rises to $130-131^{\circ}$ (Found : $\mathrm{C}=63.1$; $\mathrm{H}=6 \cdot 6 . \quad \mathrm{C}_{13} \mathrm{H}_{16} \mathrm{O}_{3} \mathrm{~N}_{2}$ requires $\mathrm{C}=62.9 ; \mathrm{H}=6.4$ per cent.).

When this acetalyl amide was warmed with alcoholic hydrogen chloride, it dissolved, and in a short time, yellow crystals of a hydrochloride commenced to separate. These were collected, ground with dilute ammonia, and the almost colourless base crystallised from alcohol, from which it separated in yellow needles melting at $247^{\circ}$. That this substance was 5-keto-4:5-dihydroindolediazine $(1: 4)$ was evident from the examination of its properties and was proved by admixture with the specimen of this substance which had previously been obtained (Part V, p. 1627), when the mixture melted at $247^{\circ}$. 
HARMINE AND HARMALINE. PART VI View Article Online

This interesting acid, prepared during the course of the present investigation, is readily obtained under the following conditions.

Phenylhydrazinoacetic acid, $\mathrm{C}_{6} \mathrm{H}_{5} \cdot \mathrm{N}\left(\mathrm{NH}_{2}\right) \mathrm{CH}_{2} \cdot \mathrm{CO}_{2} \mathrm{H}$, prepared by the method recommended by Busch (Ber., 1903, 36, 3882) and dissolved in alcohol, is mixed with the quantity of $\alpha$-ketoglutaric acid, $\mathrm{CO}_{2} \mathrm{H} \cdot \mathrm{CO} \cdot \mathrm{CH}_{2} \cdot \mathrm{CH}_{2} \cdot \mathrm{CO}_{2} \mathrm{H}$, required to yield the hydrazone, and hydrogen chloride passed until the solution is saturated with the gas, the whole being cooled during the operation. On diluting the red solution with water, a crystalline precipitate comes down, which is collected, dried, and recrystallised from alcohol, from which it separates in slender, colourless needles (Found: $\mathrm{C}=61 \cdot 6$; $\mathrm{H}=6 \cdot 0 . \quad \mathrm{C}_{17} \mathrm{H}_{19} \mathrm{O}_{6} \mathrm{~N}$ requires $\mathrm{C}=61 \cdot 3 ; \mathrm{H}=5.7$ per cent.). This diethyl ester of 2-carboxyindole-1 :3-diacetic acid decomposes at $200-202^{\circ}$, dissolves in sodium carbonate in the cold, and, when heated, develops an odour resembling that of indole. With Erhlich's reagent, it gives a bright red coloration which becomes blue on adding a drop- of nitrite, and on dilution, purple and then green; the addition of hydrochloric acid brings back the purple colour.

2-Carboxyindole-1 : 3-diacetic acid is obtained from this ester by boiling with methyl-alcoholic potassium hydroxide; water is added, the methyl alcohol removed by evaporation, and the solution acidified, when colourless needles of this tribasic acid separate; these melt at $261-263^{\circ}$ (Found : $\mathrm{C}=56 \cdot 2 ; \mathrm{H}=4 \cdot 3 . \quad \mathrm{C}_{13} \mathrm{H}_{11} \mathrm{O}_{6} \mathrm{~N}$ requires $\mathrm{C}=56 \cdot 3 ; \mathrm{H}=4.0$ per cent.).

When phenylhydrazinoacetic acid is added to an alcoholic solution of pyruvic acid and hydrogen chloride passed, some ammonium chloride separates, and dilution with water precipitates an oil which does not solidify and is extracted with ether. After hydrolysis with alcoholic potassium hydroxide, dilution with water, and removal of the alcohol by evaporation, hydrochloric acid precipitates an acid which crystallises in long, flat needles and melts at $232^{\circ}$. This acid gives a red coloration on warming with Ehrlich's reagent and is evidently 2-carboxyindole-1-acetic acid. We propose to examine this acid and also 2-carboxyindole-1 : 3 diacetic acid in more detail.

\section{Py-N-Methyl-Ind-N-propylharmine Chloride (Formula XXIII).}

Pure methylharmine (4 grams) was dissolved in a hot mixture of nitrobenzene (15 c.c.) and toluene (15 c.c.), and propyl iodide 
(5 c.c.) added. After boiling during ten minutes, the reaction, as indicated by the separation of methylpropylharmine iodide, appeared to be complete and, after cooling, the salt was collected and washed with ether. The iodide was converted to chloride by digestion on the steam-bath with water and an excess of precipitated silver chloride, and the crude salt thrown down from the filtered solution by the addition of sodium chloride and a little hydrochloric acid. The substance was then crystallised thrice from dilute hydrochloric acid and twice again from water. The crystals were collected and dried in the air, and this material lost 20.9 per cent. when dried at $125^{\circ}$ (Found: in anhydrous material, $\mathrm{C}=67.3 ; \mathrm{H}=7.0$; $\mathrm{Cl}=11 \cdot 4 . \quad \mathrm{C}_{17} \mathrm{H}_{21} \mathrm{ON}_{2} \mathrm{Cl}, 4 \cdot 5 \mathrm{H}_{2} \mathrm{O}$ requires $\mathrm{H}_{2} \mathrm{O}=21 \cdot 0$ per cent. $\mathrm{C}_{17} \mathrm{H}_{21} \mathrm{ON}_{2} \mathrm{Cl}$ requires $\mathrm{C}=67.1 ; \mathrm{H}=6.9 ; \mathrm{Cl}=11.6$ per cent.). Since the crystals lost some of their brilliancy on drying in the air, it is possible that they originally contained $5 \mathrm{H}_{2} \mathrm{O}$. This substance is referred to below as (A).

\section{Py-N-Propyl-Ind-N-methylharmine Chloride (Formula XXII).}

Harmine was quantitatively converted into its propiodide in the following manner. The base ( 4 grams) was heated with nitrobenzene (15 c.c.) until it dissolved, in a flask attached to a reflux condenser, and propyl iodide (10 c.c.) added. In a few seconds the propiodide crystallised in yellow prisms, and benzene (50 c.c.) was then added and the mixture boiled during an hour and a half. After cooling, the salt was collected and washed with benzene. The derivative crystallises very well from alcohol, in which it is sparingly soluble, in well-shaped, rectangular, prismatic needles which melt and decompose at $240^{\circ}$. Addition of sodium hydroxide to an alcoholic solution produces a yellow liquid which exhibits a striking green fluorescence.

This propiodide was converted to the corresponding chloride in the usual way by means of silver chloride, and the solution concentrated after filtration. On the addition of sodium chloride the chloride crystallised in pale yellow needles and was recrystallised from dilute brine. A hot aqueous solution was then rendered strongly alkaline by means of sodium hydroxide, and the oily yellow base extracted by warm toluene. This yellow extract, which exhibited green fluorescence, was rapidly separated and dried by warming with sticks of potassium hydroxide, and when thoroughly dry it was decanted, heated at $100^{\circ}$, and neutral methyl sulphate slowly added until the yellow colour had disappeared. A very pale yellow, crystalline substance separated almost immediately and this was collected and converted to chloride by crystallisation from dilute hydrochloric acid containing sodium chloride. Finally, 
the substance was crystallised several times from water. Airdried material lost 22.9 per cent. at $125^{\circ}$ (Found : in anhydrous material, $\quad \mathrm{C}=67 \cdot 4 ; \quad \mathrm{H}=7.0 ; \quad \mathrm{Cl}=11 \cdot 8 . \quad \mathrm{C}_{17} \mathrm{H}_{21} \mathrm{ON}_{2} \mathrm{Cl}, 5 \mathrm{H}_{2} \mathrm{O}$ requires $\mathrm{H}_{2} \mathrm{O}=22 \cdot 8$ per cent. $\mathrm{C}_{17} \mathrm{H}_{21} \mathrm{ON}_{2} \mathrm{Cl}$ requires $\mathrm{C}=67 \cdot 1$; $\mathrm{H}=6.9 ; \quad \mathrm{Cl}=11.6$ per cent.). This substance is termed (B) in the comparison of properties of the two isomeric salts, which may now be made.

(A) melts at $130^{\circ}$, whereas (B) melts at $100^{\circ}$, and after resolidification (A) melts at $240^{\circ}$ with decomposition whilst (B) still melts at about $100-110^{\circ}$. Both salts are hygroscopic when anhydrous. The isomerides are readily soluble in water, but (A) is much the more sparingly soluble of the two. The brilliant blue fluorescence of the aqueous solutions are identical. If to separate portions of an aqueous solution of (A) hydrochloric acid, nitric acid, sulphuric acid, or oxalic acid is added, an immediate crystalline precipitate of the corresponding salt is produced. These salts crystallise in small, colourless needles. With a solution of (B) of the same concentration, the salts are not immediately precipitated, but separate after some time in much larger slender needles. On the addition of potassium hydroxide to a solution of (A), there is produced a yellow liquid and a precipitate of yellow needles soluble in excess of water. With (B), however, the precipitate is a yellow oil also soluble in excess of water. A proof of the fact that (A) and (B) are different substances is afforded by the behaviour with sulphuric acid. Both give a yellow solution which exhibits bright green fluorescence and on warming becomes colourless and exhibits blue fluorescence. On diluting the solution of (A) in warm sulphuric acid, there is an immediate precipitate of a sulphonic acid. This substance is very sparingly soluble and crystallises in very small, colourless needles. (B), treated in the same way, never gives an immediate precipitate, but after some days the solution deposited crystals in this case also. These are short, slender, colourless needless similar to those obtained from (A), but exhibiting a greater tendency to form clusters. The sulphonic acid from (B), if used to inoculate subsequent preparations, produces an immediate crystallisation, but that from (A) has no such effect. Both (A) and (B) give very sparingly soluble mercurichlorides crystallising in pale yellow needles.

(A) gives a more flocculent, orange-yellow precipitate with potassium dichromate than does $(B)$, whereas the buff ferrichloride from (B) is more flocculent than that from (A). Both salts give a green solution in concentrated nitric acid and a curious green oil when shaken with benzoyl chloride and aqueous potassium hydroxide. 
Dimethylharminesulphonic Acid,

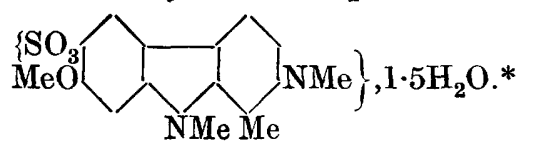

In Part III (p. 950), the very sparingly soluble methylharminesulphonic acid was described, and it was tentatively suggested that the substance might be an $N$-sulphonic acid derivative. In view of the fact that dimethylharmine chloride and the two methylpropylharmine chlorides yield similar derivatives, it must now be assumed that the sulphonic acid group enters the benzene nucleus. Dimethylharmine chloride ( 2 grams) was dissolved in sulphuric acid (10 c.c.). The yellow solution had a most brilliant green fluorescence, but the colour disappeared and the fluorescence changed to blue-violet on warming for a few seconds on the steam-bath. The mixture was added to excess of water, when an immediate precipitate of a very sparingly soluble substance was obtained. This was collected, washed with hot water, and dried at $110^{\circ}$ (Found : $\mathrm{C}=52 \cdot 0 ; \quad \mathrm{H}=5 \cdot 7 ; \quad \mathrm{S}=9 \cdot 2 . \quad \mathrm{C}_{15} \mathrm{H}_{16} \mathrm{O}_{4} \mathrm{~N}_{2} \mathrm{~S}, 1 \cdot 5 \mathrm{H}_{2} \mathrm{O} \quad$ requires $\mathrm{C}=52.0 ; \mathrm{H}=5.5 ; \mathrm{S}=9 \cdot 2$ per cent.). This substance crystallises from a very large volume of water in slender, microscopic needles. It is insoluble in sodium carbonate and only dissolves in very dilute aqueous sodium hydroxide on heating

Dyson Perrins Laboratory, Oxford.

Chemistry Regearch Laboratory,

Untted College of St. Salvator and St. Leonard, UNIVERSITY OF ST. ANDREWS.

Research Laboratory of the Royal College of Physicians, EDINBURGH.

[Received, August 1st, 1922.] 